\title{
Detection of Plasmodium Falciparum in Laboratory and Naturally Infected Mosquitoes Using Near- infrared Spectroscopy
}

Dari F. Da ( $\sim$ dafrenick@yahoo.fr)

Institut de Recherche en Sciences de la Santé, Direction Régionale, 399 avenue de la liberté, Bobo Dioulasso 01 BP 54501

\section{Ruth McCabe}

MRC Centre for Global Infectious Disease Analysis, Infectious Disease Epidemiology, Imperial College London

\section{Bernard M. Somé}

Institut de Recherche en Sciences de la Santé, Direction Régionale, 399 avenue de la liberté, Bobo

Dioulasso 01 BP 54501

\section{Pedro M. Esperança}

MRC Centre for Global Infectious Disease Analysis, Infectious Disease Epidemiology, Imperial College London

\section{Kasia Sala}

Department of Life Sciences, Imperial College London, Sir Alexander Fleming Building, Exhibition Road, South Kensington

\section{Josua Blight}

Department of Life Sciences, Imperial College London, Sir Alexander Fleming Building, Exhibition Road, South Kensington

Andrew M. Blagborough

Division of Microbiology and Parasitology, Department of Pathology, Cambridge University

Floyd Dowell

Stored Product Insect and Engineering Research Unit, United States Department of

Agriculture/Agricultural Research Services, Center for Grain and Animal Health Research, Manhattan, Kansas

\section{Serge R. Yerbanga}

Institut de Recherche en Sciences de la Santé, Direction Régionale, 399 avenue de la liberté, Bobo Dioulasso 01 BP 54501

\section{Thierry Lefèvre}

MIVEGEC, Montpellier University, IRD, CNRS, Montpellier

\section{Karine Mouline}

MIVEGEC, Montpellier University, IRD, CNRS, Montpellier 


\section{Roch K. Dabiré}

Institut de Recherche en Sciences de la Santé, Direction Régionale, 399 avenue de la liberté, Bobo Dioulasso 01 BP 54501

\section{Thomas S. Churcher}

MRC Centre for Global Infectious Disease Analysis, Infectious Disease Epidemiology, Imperial College London

\section{Research Article}

Keywords: Plasmodium falciparum, Near-infrared spectroscopy (NIRS), mosquitoes, Mosquito-borne diseases

Posted Date: February 10th, 2021

DOI: https://doi.org/10.21203/rs.3.rs-178309/v1

License: (9) This work is licensed under a Creative Commons Attribution 4.0 International License. Read Full License 


\title{
Title: Detection of Plasmodium falciparum in laboratory and naturally infected mosquitoes using near-infrared spectroscopy
}

\author{
Authors: \\ Dari F. Da ${ }^{1 *}$, Ruth McCabe $^{2}$, Bernard M. Somé ${ }^{1}$, Pedro M. Esperança ${ }^{2}$, Kasia Sala ${ }^{3,4}$, Josua \\ Blight $^{4}$, Andrew M. Blagborough ${ }^{3}$, Floyd Dowell ${ }^{5}$, Serge R. Yerbanga ${ }^{1}$, Thierry Lefèvre ${ }^{6,7,8}$, \\ Karine Mouline ${ }^{6,7}$, Roch K. Dabiré ${ }^{1,7}$, Thomas S. Churcher ${ }^{2}$
}

\begin{abstract}
Affiliations:
${ }^{1}$ Institut de Recherche en Sciences de la Santé, Direction Régionale, 399 avenue de la liberté, Bobo Dioulasso 01 BP 545 01, Burkina Faso

${ }^{2}$ MRC Centre for Global Infectious Disease Analysis, Infectious Disease Epidemiology, Imperial College London, London W2 1PG, UK.

${ }^{3}$ Division of Microbiology and Parasitology, Department of Pathology, Cambridge University, Cambridge CB2 1QP, UK.

${ }^{4}$ Department of Life Sciences, Imperial College London, Sir Alexander Fleming Building, Exhibition Road, South Kensington, London, UK.

${ }^{5}$ Stored Product Insect and Engineering Research Unit, United States Department of Agriculture/Agricultural Research Services, Center for Grain and Animal Health Research, Manhattan, Kansas, USA.

${ }^{6}$ MIVEGEC, Montpellier University, IRD, CNRS, Montpellier, France.

${ }^{7}$ Laboratoire Mixte International sur les Vecteurs (LAMIVECT), Bobo Dioulasso, Burkina Faso

${ }^{8}$ Centre de Recherche en Écologie et Évolution de la Santé (CREES), Montpellier, France.

*To whom correspondence should be addressed: dafrenick@yahoo.fr

\section{Author contact:}

Dari F. Da: dafrenick@yahoo.fr ; Ruth McCabe: ruth.mccabe17@imperial.ac.uk ; Bernard M. Somé: bernardsilome40@gmail.com ; Pedro M. Esperança: pedro.esp88@gmail.com ; Kasia Sala: katarzyna.sala@crick.ac.uk ; Josua Blight: josua.blight09@imperial.ac.uk ; Andrew M. Blagborough: amb283@cam.ac.uk; Floyd Dowell: floyd.dowell@gmail.com ; Serge R. Yerbanga: yrserge@yahoo.fr ; Thierry Lefèvre: thierry.lefevre@ird.fr ; Karine Mouline : karine.mouline@ird.fr ; Roch K. Dabiré: dabireroch@gmail.com ; Thomas S. Churcher: thomas.churcher@imperial.ac.uk
\end{abstract}

One Sentence Summary: Near-infrared spectroscopy successfully determines malaria infection in laboratory-reared mosquitoes but detection in wild mosquitoes remains poor. 


\begin{abstract}
:
There is an urgent need for high throughput, affordable methods of detecting pathogens inside insect vectors to facilitate surveillance. Near-infrared spectroscopy (NIRS) has shown promise to detect arbovirus and malaria in the laboratory but has not been evaluated in field conditions. Here we investigate the ability of NIRS to identify Plasmodium falciparum in Anopheles coluzzii mosquitoes. NIRS models trained on laboratory-reared mosquitoes infected with wild malaria parasites can detect the parasite in comparable mosquitoes with moderate accuracy though fails to detect oocysts or sporozoites in naturally infected field caught mosquitoes. Models trained on field mosquitoes were unable to predict the infection status of other field mosquitoes. Restricting analyses to mosquitoes of uninfectious and highly-infectious status did improve predictions suggesting sensitivity and specificity may be better in mosquitoes with higher numbers of parasites. Detection of infection appears restricted to homogenous groups of mosquitoes diminishing NIRS utility for detecting malaria within mosquitoes.
\end{abstract}

\title{
Introduction
}

Mosquito-borne diseases continue to cause widespread suffering world-wide. Malaria cases are thought to have risen in the last few years following two decades of decline ${ }^{1}$ whilst the public health impact of arboviruses such as dengue, chikungunya and zika continues to increase ${ }^{2}$. Killing the mosquito vector is the most effective current method for controlling these diseases ${ }^{3}$ and it is important to monitor infection in local mosquito populations to understand the efficacy of control interventions, track disease trends and provide warnings of outbreaks. Entomological monitoring is costly and time consuming. The short life-expectancy of mosquitoes means that typically fewer than $5 \%$ of vectors are infectious even in highly endemic regions ${ }^{4}$. This means that high number of insects need to be tested to generate reliable estimates. Unfortunately, there are no cheap and easy-to-use methods of detecting pathogens in mosquitoes. In malaria, the presence of infectious sporozoites is determined either by manual salivary gland dissection using a microscope or through molecular methods such as PCR (polymerase chain reaction) or ELISA (enzyme-linked immunosorbent assay) ${ }^{5-7}$. All these techniques are laborious and are therefore costly for large sample size whilst PCR also requires well-equipped laboratories and expensive reagents. 
Near-infrared spectroscopy (NIRS) is a fast, non-destructive and reagent-free scanning technique which has been shown to detect mosquitoes infected with rodent models of malaria ${ }^{8}$, laboratory strains of human malaria ${ }^{9}$, dengue, zika ${ }^{10}$ and the endosymbiont Wolbachia bacteria ${ }^{11}$. Mosquitoes are scanned at different wavelengths in the near-infrared region of the electromagnetic spectrum and a chemometric model is used to convert spectra into estimates of pathogen prevalence. All previous NIRS infection works have been conducted on laboratory reared mosquitoes of similar age and using laboratory strains of pathogen. The accuracy of these diagnostics has been evaluated on a sub-set of the same group of mosquitoes, which is likely to overestimate sensitivity and specificity. There is also evidence that the technique may lose accuracy when there is more diverse field derived parasites and mosquitoes ${ }^{12}$. Here we evaluate the ability of NIRS to determine Anopheles coluzzii infection status with wild Plasmodium falciparum isolates circulating in Burkina Faso. This is initially conducted with laboratory-reared Anopheles before evaluating the ability of the models to detect the parasite in wild caught mosquitoes infected naturally in the field. It is unclear whether NIRS is detecting the presence of parasite biomass or a physiological change in the mosquito. Here we devise a comprehensive set of experiments which would enable the differentiation of mosquitoes which (i) have fed on malaria infected blood, (ii) are infected with oocyst life-stages (visible in Burkina Faso from 311 days from infection) ${ }^{13}$ and (iii) are infectious with salivary gland sporozoites. Sporozoites are the most epidemiologically important parasite life stage although evaluation of control interventions might be easier with earlier life-stages which have a higher prevalence in wild mosquito populations and therefore require lower number of mosquitoes to generate sufficiently precise estimates.

\section{Results}

Wild parasites inside laboratory-reared mosquitoes.

NIRS can identify mosquitoes infected and infectious with wild malaria parasites with relatively high accuracy. A total of 2452 An. coluzzii mosquitoes of ages ranging from 3 to 27 days were used to train the model (Table 1). Overall within-sample accuracy, defined as the percentage of mosquitoes correctly classified, for detecting sporozoite positive mosquitoes (uninfectious vs infectious) was $73 \%$ (sensitivity $=74 \%$, specificity $=72 \%$, Fig. 1, Table 2). NIRS was also able to differentiate between uninfected mosquitoes and those with either oocysts or sporozoites 
(uninfected vs infected), though with slightly lower accuracy (accuracy=71\%, sensitivity=71\%, specificity $=70 \%$, Fig. S1). Accuracy was similar for mosquitoes infected on Day 3 or Day 6 after emergence (accuracy $=74 \%$ and accuracy $=72 \%$, respectively, for uninfectious vs infectious; accuracy $=73 \%$ and accuracy $=72 \%$, respectively, for uninfected vs infected).

It is unclear whether NIRS is detecting parasite biomass or some metabolic or physiological response of the mosquito. NIRS had relatively poor accuracy differentiating between uninfected/uninfectious mosquitoes fed on infectious blood and blood from infectious individuals inactivated through heat treatment (balancing for mosquito age between groups, accuracy $=64 \%$ ). This suggests the presence of infectious gametocytes is not initiating an immunological response subsequently detected by NIRS or that NIRS is directly detecting developing alive parasite.

Wild parasites in wild mosquitoes using models trained on laboratory mosquitoes.

Models trained on laboratory-reared mosquitoes infected with wild parasites were unable to predict infection status of wild caught mosquitoes. Overall out-of-sample accuracy for detecting wild caught sporozoite positive mosquitoes (uninfectious vs infectious) using the model with best within-sample accuracy was accuracy $=50 \%$ (sensitivity $=56 \%$, specificity $=44 \%$ ). Varying the machine learning method to reduce overfitting improves accuracy (Table 2) though predictions are still very poor (accuracy $=52 \%$, sensitivity $=52 \%$, specificity $=51 \%$ ).

\section{Wild parasites in wild mosquitoes using models trained on wild mosquitoes}

To determine whether there is any difference in the spectra from infectious and uninfectious mosquitoes models were trained on wild-caught $A n$. coluzzii mosquitoes alone. NIRS was unable to differentiate infectious or infected-infectious mosquitoes with any accuracy (accuracy of 51\% and $51 \%$, respectively). Examining mosquitoes from the same village did not substantially improve within- or out-of-sample predictions (Table 2). 

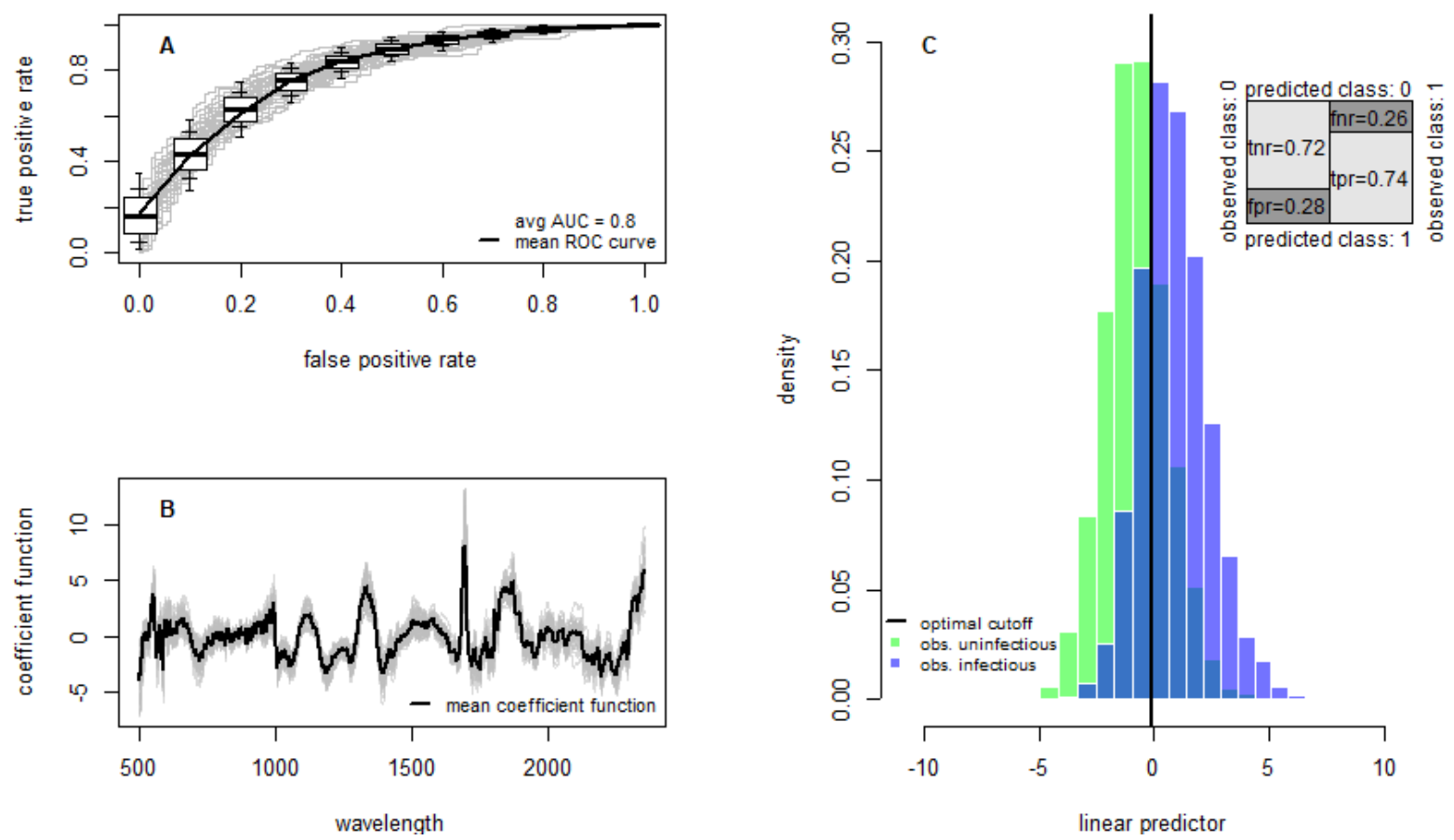

Fig. 1. The ability of NIRS to predict laboratory-reared mosquitoes infectious with wild parasites. All models were trained on sporozoite positive and sporozoite negative laboratory reared mosquitoes using all the data presented in Table 1. (A) The receiver operating characteristic (ROC) curve for the best-fit model showing the false positive and true positive rates achievable for different classification probability thresholds whilst the overall performance is given by the average area under the ROC curve (AUC). A perfect model with $100 \%$ sensitivity and specificity would be in the top left corner (coordinates 0,1 ). Solid line shows the average ROC curve with boxplots showing the variability for 50 randomizations of the training, validation and testing datasets (with box edges, inner and outer whiskers showing 25 th/75th, 15th/85th and 5th/95th percentiles, respectively; and the black line inside the box showing the median/50th-percentile). (B) Coefficient functions for the best fit model for each of the 50 dataset randomizations (grey lines) and the corresponding average (black line). (C) The histogram of the estimated linear predictor for the test mosquitoes, color of the bars indicates the true class, shows the model's ability to separate the two groups of mosquitoes. The vertical black line indicates the optimum threshold for classifying mosquitoes as infectious or not. The shaded area where the two distributions overlap corresponds to misclassified test observations - false negatives to the left and false positives to the right of the optimal classification threshold. The confusion matrix (inset) shows the different error rates: tnr, true negative rate (specificity); fnr, false negative rate; fpr, false positive rate; and tpr, true positive rate (sensitivity). 
Table 1. The number of laboratory and field mosquitoes analyzed.

All data were Anopheles coluzzii mosquitoes infected with wild strains of Plasmodium falciparum.

\begin{tabular}{|c|c|c|c|c|c|c|c|c|c|c|c|c|c|c|}
\hline & \multicolumn{11}{|c|}{ Days since feeding (laboratory-reared mosquitoes) } & \multicolumn{3}{|c|}{ Wild caught mosquitoes } \\
\hline & 3 & 5 & 7 & 9 & 11 & 13 & 15 & 17 & 19 & 21 & Total & Longo & Klesso & Total \\
\hline \multicolumn{15}{|c|}{ Unexposed to infectious gametocytes } \\
\hline $\begin{array}{l}\text { Inactivated } \\
\text { blood }\end{array}$ & 100 & 100 & 140 & 100 & 100 & 100 & 109 & 90 & 45 & 20 & 904 & NA & NA & NA \\
\hline \multicolumn{15}{|c|}{ Fed infectious gametocytes } \\
\hline Uninfected & 147 & 110 & 106 & 75 & 73 & 70 & 58 & 40 & 39 & 1 & 719 & $2445^{\pi}$ & 80 & 2525 \\
\hline $\begin{array}{l}\text { Infected } \\
\text { (oocysts) }\end{array}$ & 45 & 88 & 91 & 112 & 104 & 102 & 101 & 90 & 66 & 30 & 829 & 387 & 25 & 412 \\
\hline $\begin{array}{l}\text { Infectious }^{*} \\
\text { (sporozoites) }\end{array}$ & 0 & 0 & 0 & 51 & 92 & 102 & 101 & 90 & 66 & 30 & 532 & 302 & 21 & 323 \\
\hline Total & 292 & 298 & 337 & 287 & 277 & 272 & 268 & 220 & 220 & 51 & 2452 & 2832 & 105 & 2937 \\
\hline
\end{tabular}

II Blood source unknown as mosquitoes were collected potentially exposed

*All infectious mosquitoes were classified as also infected (whether or not oocysts were visible) 
Table 2. Summary of overall accuracy of the different NIRS models for predicting presence of sporozoites.

Models were trained on either laboratory or field mosquitoes, either all mosquitoes grouped together (all) or separately for mosquitoes from the villages of Longo (V1) or Klesso (V2). The number of PLS components $(Q)$ is presented alongside overall models accuracy (the percentage of mosquitoes correctly classified), the true positive rate (TPR) and false positive rate (FPR). This is shown for either within sample accuracy (where the same group of mosquitoes were used to train/validate and test the model) or out-of-sample accuracy (where a different group of wild caught mosquitoes were used). For within-sample accuracy different individual mosquitoes were used to train, validate and test the model though out-of-sample evaluation provides a more robust test as different groups (i.e. laboratory vs field or different field locations) were used to assess accuracy. Two different models are presented for out-of-sample accuracy, either the most accurate either within-sample or out-of-sample (which tend to be more generalizable and have lower numbers of components, denoted $Q$ ).

\begin{tabular}{|c|c|c|c|c|c|c|c|c|c|c|c|c|}
\hline \multirow[b]{3}{*}{ Model trained on } & \multicolumn{4}{|c|}{ Within-sample accuracy } & \multirow[b]{3}{*}{ Model predicting } & \multicolumn{7}{|c|}{ Out-of-sample accuracy } \\
\hline & \multirow[b]{2}{*}{$\begin{array}{c}\text { Best } \\
\text { model } \\
(\mathrm{Q})\end{array}$} & \multirow[b]{2}{*}{$\begin{array}{l}\text { Accuracy } \\
\text { (std error) }\end{array}$} & \multirow[b]{2}{*}{ TPR } & \multirow[b]{2}{*}{ FPR } & & \multicolumn{3}{|c|}{ Best within-sample model } & \multicolumn{4}{|c|}{ Best out-of-sample model } \\
\hline & & & & & & $\begin{array}{l}\text { Accuracy } \\
\text { (std error) }\end{array}$ & TPR & FPR & $\begin{array}{c}\text { Best } \\
\text { model }(\mathrm{Q})\end{array}$ & $\begin{array}{c}\text { Accuracy } \\
\text { (std } \\
\text { error) }\end{array}$ & TPR & FPR \\
\hline Laboratory mosquitoes & $\begin{array}{c}\text { GLM } \\
(11)\end{array}$ & $\begin{array}{c}73 \% \\
(0.02)\end{array}$ & $74 \%$ & $72 \%$ & Field mosquitoes (all) & $\begin{array}{c}50 \% \\
(0.01)\end{array}$ & $56 \%$ & $44 \%$ & $\begin{array}{c}\text { fsGLM } \\
\text { (4) }\end{array}$ & $\begin{array}{c}52 \% \\
(0.007)\end{array}$ & $52 \%$ & $51 \%$ \\
\hline Field mosquitoes (all) & $\begin{array}{l}\text { fsGLM } \\
\text { (2) }\end{array}$ & $\begin{array}{l}51 \% \\
(0.04)\end{array}$ & $65 \%$ & $37 \%$ & & NA & NA & NA & NA & NA & NA & NA \\
\hline Field mosquitoes (V1) & $\begin{array}{c}\text { pGLM } \\
\text { (2) }\end{array}$ & $\begin{array}{l}51 \% \\
(0.04)\end{array}$ & $57 \%$ & $46 \%$ & Field mosquitoes (V2) & $\begin{array}{l}51 \% \\
(0.05)\end{array}$ & $58 \%$ & $45 \%$ & $\begin{array}{c}\text { fpGLM } \\
\text { (2) }\end{array}$ & $\begin{array}{l}52 \% \\
(0.05)\end{array}$ & $60 \%$ & $43 \%$ \\
\hline Field mosquitoes (V2) & $\begin{array}{c}\text { fpGLM } \\
\text { (5) }\end{array}$ & $\begin{array}{l}47 \% \\
(0.1)\end{array}$ & $28 \%$ & $64 \%$ & Field mosquitoes (V1) & $\begin{array}{l}51 \% \\
(0.02)\end{array}$ & $30 \%$ & $71 \%$ & $\begin{array}{c}\text { fspGLM } \\
\text { (5) }\end{array}$ & $\begin{array}{l}51 \% \\
(0.02)\end{array}$ & $39 \%$ & $63 \%$ \\
\hline
\end{tabular}




\section{Impact of mosquito age on detection}

NIRS can differentiate the age of laboratory-reared mosquitoes with high accuracy ${ }^{14,15}$. Previous laboratory studies investigating infection have only used single aged mosquitoes ${ }^{9,16}$, dissecting all mosquitoes on the same day when oocysts or sporozoites are visible ${ }^{17}$. To generate more robust results a range of different aged mosquitoes were compared here which could in part account for somewhat lower accuracy than previous studies. Field mosquitoes will have to be greater than 13 days old if an extrinsic incubation period of 10 days is assumed. The inability of NIRS to detect wild infectious mosquitoes could be associated with the informative region of the spectra interacting with wavelengths that change with mosquito age. To test this hypothesis models were trained on laboratory-reared mosquitoes using a two-step process. Firstly, it was determined whether an individual mosquito was >13 days old which the model achieved with high accuracy (within-sample accuracy $=84 \%$ ). Secondly, older mosquitoes were then used to train the model for infectiousness which was again achieved with high accuracy (within-sample accuracy $=76 \%$ ). Nevertheless, repeating the process on field mosquitoes failed to improve model predictions which suggesting that different age distributions of mosquitoes cannot explain the contrasting results.

\section{Impact of parasite intensity on diagnosis}

It is likely that the number of sporozoites in wild caught mosquitoes may be substantially lower than those infected through a direct membrane feeding assay. The mean number of oocysts per oocyst-positive mosquito was 8.39 in the laboratory experiments and 3.05 recorded from wild caught mosquitoes. This may cause the spectra from laboratory and field-reared to differ. To investigate this the models trained on field mosquitoes were rerun comparing uninfectious mosquitoes with those infectious mosquitoes with $>20$ sporozoites per mosquito (as determined by quantitative PCR). Accuracy of the models does improve suggesting parasite intensity might be a contributing factor, though the predictive ability is still poor (accuracy $=56 \%$, sensitivity $=55 \%$, specificity $=57 \%$, Fig. 2), and the number of naturally-infected mosquitoes with high sporozoite loads available to fit the model was relatively low (37 An. coluzzii). Similarly improved results were seen when models trained on laboratory mosquitoes were used to predict 
wild caught mosquitoes which were either uninfectious or highly infectious (accuracy=64\%, sensitivity $=67 \%$, specificity $=61 \%$, Fig. S2).
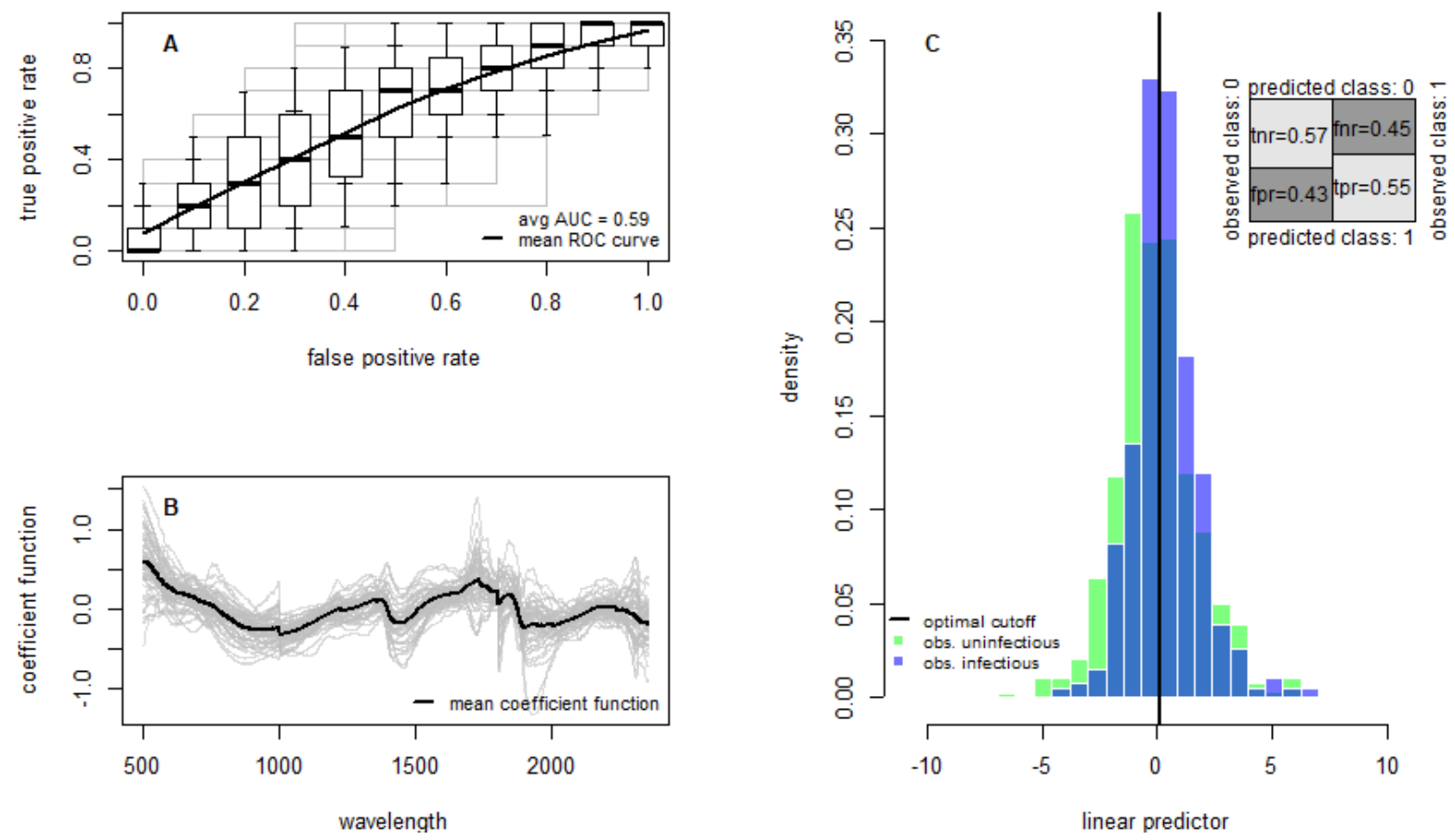

Fig. 2. The ability of NIRS to predict field caught mosquitoes with high number of sporozoites. All models were trained using mosquitoes infected in the wild and were either sporozoite positive mosquitoes with $>20$ sporozoite per Anopheles (20 gene copy number as defined by qPCR) or sporozoite negative mosquitoes (Table 1) (A) The ROC curve for the bestfit model showing the false positive and true positive rates achievable for different classification probability thresholds whilst the overall performance is given by the AUC. Solid line shows the average ROC curve with boxplots showing the variability for 50 randomizations of the training, validation and testing datasets (with box edges, inner and outer whiskers showing $25 \mathrm{th} / 75 \mathrm{th}$, 15 th/85th and 5th/95th percentiles, respectively; and the black line inside the box showing the median/50th-percentile). (B) Coefficient functions for the best fit model for each of the 50 dataset randomizations (grey lines) and the corresponding average (black line). (C) The histogram of the estimated linear predictor for the test mosquitoes, color of the bars indicates the true class, shows the model's ability to separate the two groups of mosquitoes. The vertical black line indicates the optimum threshold for classifying mosquitoes as infectious or not. The shaded area where the two distributions overlap corresponds to misclassified test observations - false negatives to the left and false positives to the right of the optimal classification threshold. The confusion matrix (inset) shows the different error rates: tnr, true negative rate (specificity); fnr, false negative rate; fpr, false positive rate; and tpr, true positive rate (sensitivity). 


\section{Discussion}

We show that NIRS can detect the presence of natural malaria parasites in laboratory-reared mosquitoes but cannot differentiate between uninfectious or infectious wild mosquitoes with any accuracy. Similar poor accuracy was seen for wild mosquitoes when models were trained on either laboratory-reared or wild mosquitoes. The failure of NIRS to differentiate between infectious and uninfectious wild mosquitoes suggests the technique is unlikely to be able to detect sporozoites in the field. A large number of mosquitoes (nearly 3000 in total, with over 300 mosquitoes sporozoite positive) and a variety chemometric approaches ${ }^{16}$ were used in the analysis. Though it cannot be discounted that larger sample sizes and different machine learning methods ${ }^{18,19}$ may improve predictions, the lack of differentiation between groups suggests substantial improvements are unlikely.

The reason why the technique works well when mosquitoes are infected in the laboratory but not naturally in the field remain unclear. In the field, sporozoite positive mosquitoes are likely to be older than the average mosquito due to the relatively long extrinsic incubation period of the parasite. Previous laboratory studies have only compared older mosquitoes of the same age so regions of the spectrum informative for age could also be indicative of infection status. Using the model to predict mosquito age first and then evaluating infectious status in the older mosquitoes did not improve identification of sporozoite positive wild mosquitoes. This would suggest that differences in the age distribution may not be the cause of the discrepancy though the ability of NIRS to evaluate the age of wild caught mosquitoes has not currently been evaluated in the field. Indeed, the inability of NIRS to detect sporozoites, which will on average be in older mosquitoes, questions the ability of the technique to determine age in field populations, though further investigation is necessary. Beside age heterogeneity, other factors including larval breeding site diversity, blood-meal and sugar sources, physiological and nutritional status may explain why NIRS is poorly able to determine the infection status of wild mosquitoes.

Results show that direct membrane feeding experiments on average generate substantially higher intensity infections than that observed in wild mosquitoes. This difference in the quantity of parasite biomass could be a factor contributing to the lower accuracy in field mosquitoes. This hypothesis is supported by the accuracy improving when comparing uninfectious vs highly infectious wild infected mosquitoes, though only 37 mosquitoes were identified with more than 
20 sporozoites in the salivary gland were identified by qPCR so further improvements might be seen with larger sample sizes. It is unclear whether in the laboratory, NIRS is detecting parasite biomass or an immunological response to the parasite. The lack of differences in the spectra of uninfected mosquitoes fed infectious blood or blood from the same individuals heat treated to kill gametocytes suggests that this life-stage is not initiating the immunological reaction, though it cannot be discounted that infertile gametocytes could still initiate the response. Here we were able to identify infected (positive for oocysts and sporozoites) and infectious (positive for sporozoites) with similar accuracy. This is consistent with previous work using laboratory strains of the same parasite which was able to identify the presence of both oocysts and sporozoites ${ }^{9}$. Promising laboratory results and the ease and utility of the technique means that NIRS could substantially improve monitoring of mosquito populations in the wild. The technique has the potential to determine mosquito species and age at the same time though unfortunately the evidence presented here suggests that it cannot detect whether a mosquito contains the malaria parasite as well. The need to examine large numbers of mosquitoes and the high cost of molecular methods means that there may be some utility in triaging mosquitoes using NIRS before suspected infections are confirmed using other methods. NIRS and other spectrometry methods such as mid-infrared spectromotery ${ }^{20,21}$ could still substantially revolutionize the monitoring of wild mosquito populations. Nevertheless, the work presented here joins a growing body of evidence that ${ }^{12}$ highlights the problems associated with transferring these potentially useful entomological tools from the laboratory to the field.

\section{Materials and Methods}

\section{$\underline{\text { Experimental Design }}$}

A comprehensive set of laboratory experiments were designed to understand the sensitivity and specificity of NIRS to detect different life-stages of the parasite inside laboratory-reared and field mosquitoes. Previous work has shown that NIRS can detect oocysts and sporozoites 7 and 14 days post infection, respectively ${ }^{9}$, in laboratory strains of the parasite and mosquito. Nevertheless, it is unclear whether it is detecting parasite biomass directly or a physiological change in infected mosquitoes which could be initiated by earlier life-stages (for example the ookinete stage which penetrates the mosquito mid-gut wall). To disentangle the possible cause 
and understand how the likelihood of detecting the parasite changes with mosquito age and time since infection mosquitoes are fed infectious and non-infectious blood on either day 3 or 6 following emergence and scanned every other day until all mosquitoes have died. Ethical approval was gained from Imperial College Research Ethics Committee (18IC4859) and “Comité d'Ethique Institutionnel pour la Recherche en Sciences de la Santé, Burkina Faso" (clearance A018-2017/CEIRES). The protocols and associated procedures were conformed to the current international legislation and recommendations, including bioethics specificities in Burkina Faso.

\section{$\underline{\text { Laboratory mosquitoes }}$}

Anopheles coluzzii females were exposed to malaria using a direct membrane feeding assays (DMFAs). Blood with Plasmodium falciparum gametocytes was obtained from three volunteer children (aged 5-11 years) naturally infected with malaria living in villages surrounding BoboDioulasso, after obtaining their parent/guardian's informed consent. Stratified gametocyte densities (low, medium and high gametocytemia) were required expecting to generate various infection level in experimental groups of Anopheles). For each experiment replicate, venous blood was drawn in heparinized tubes and immediately centrifuged at 3,000 rpm for 3 minutes to remove the supernatant, replacing it with non-immune serum from a European $\mathrm{AB}+$ donor to increase infection rates. Three and six days old female mosquitoes from an outbred Anopheles coluzzii local colony were starved overnight and fed on the blood mixture through pre-warmed membrane feeders for 30 minutes. Fully fed females were sorted and maintained in cages at $28{ }^{\circ} \mathrm{C} \pm 2,80 \% \pm 05 \mathrm{RH}$ with $10 \%$ glucose solution. From day 3-21 post-blood meal mosquitoes were killed by chloroform vapor and immediately scanned. Mosquitoes killed 3-11 days from blood-feeding were immediately dissected using a light microscope and the number of oocysts on the midgut were counted. Mosquitoes killed 9-21 days were also assessed for salivary gland sporozoites using quantitative PCR ${ }^{22}$. A control group of uninfected and uninfectious Anopheles

were generated by feeding some females with gametocytes inactivated blood ${ }^{23}$ by heating a sample of the same blood used to infect mosquitoes at $45^{\circ} \mathrm{C}$ for 20 minutes to kill all gametocytes to provide an uninfectious control feed. 


\section{Wild mosquitoes}

Mosquitoes were caught in the houses of two villages in the Bobo-Dioulasso region of Burkina Faso. The villages of Longo and Klesso were $120 \mathrm{~km}$ apart to allow the robustness of the method over space to be assessed. Wild mosquitoes were caught by the technicians using a mouth aspirator in the living room of human dwellings early in the morning ${ }^{24}$ and transferred to the laboratory. They were maintained in cages $(30 \times 30 \times 30 \mathrm{~cm})$ in laboratory conditions $\left(28^{\circ} \mathrm{C} \pm 2\right.$, $80 \% \pm 05 \mathrm{RH}$ with $10 \%$ glucose solution) during 3 or 7 days periods before the next step. At these indicated periods, the Anopheles females were scanned using the spectrometer and their midgut were immediately dissected (via microscopy) to determine oocyst prevalence. The remaining carcass (head-thorax) were molecular analyzed for Anopheles species identification ${ }^{25}$ and sporozoites detection in salivary glands ${ }^{22}$. Only Anopheles coluzzii mosquitoes identified by PCR were included for statistical analysis for NIRS P. falciparum infection detection. All mosquitoes identified as being $P$. falciparum positive were further analysed through quantitative PCR to determine sporozoite intensity. QPCR analysis of gDNA was used to quantify the gene copy number in the mosquitoes. Analysis was performed in triplicate in 10ul reaction using BioRad SSO Advanced Universal Sybr Green Supermix (BioRad, 1725272) and the Roche LightCycler 480. Primers were designed to amplify fragment of Plasmodium falciparum HSP70 gene with the following sequences: forward primer GAGGTATGCCCGGTGGAATG; reversed primer CTGTTGGTCCACTTCCAGCT. Reactions were 40 cycles using following conditions: initial denaturation for $3 \mathrm{~min}$ at $95^{\circ} \mathrm{C}$, and 40 cycles of $10 \mathrm{sec}$ denaturation at $95^{\circ} \mathrm{C}$ and $20 \mathrm{sec}$ amplification at $60^{\circ} \mathrm{C}$. The number of HSP70 gene copies in gDNA extracted from mosquito was calculated from their respective $\mathrm{Ct}$ value based on plasmid standard curve. The standard curve was generated from serial dilutions of a plasmid pGEMPfHSP70 containing Plasmodium falciparum HSP70 gene. Mosquitoes with over 20 gene copy numbers were classified as being highly infectious.

\section{Mosquito scanning}

Mosquitoes were killed with chloroform vapor and scanned using a LabSpec4 Standard-Res i (standard resolution, integrated light source) near-infrared spectrometer and a bifurcated reflectance probe mounted $2 \mathrm{~mm}$ from a spectralon white reference panel (ASD Inc., 
Westborough, Massachusetts , USA). Absorbance at 2151 wavelengths from 350-2500 nanometers of the electromagnetic spectrum was recorded using RS3 spectral acquisition software (ASD Inc., Westborough, Massachusetts, USA [17]) which averaged spectra from 20 scans. All mosquitoes were scanned on both sides centering the light probe on the head and thorax region.

\section{$\underline{\text { Statistical Analysis }}$}

Machine learning methods were used to construct binomial logistic regression models using maximum likelihood. The mean of the two spectra from each mosquito were used in the analysis. Spectra were then trimmed to values corresponding to $500-2350 \mathrm{~nm}$ to remove the excess noise arising from the sensitivity of the spectrometer at the ends of the near-infrared range ${ }^{26}$. These spectra were analysed using partial least squares regression (PLS), a statistical technique utilising the covariance between the spectra and infection status in order to extract the most informative elements within a much smaller dimension. This method generates different numbers of principal components which are linearly independent and used as the explanatory variables in the regression model. An upper limit of 20 components was enforced, with the optimal number of components being determined via ordinary cross-validation.

In conjunction with the use of PLS, three additional techniques were used to further improve model generalisability through ensuring as smooth a coefficient function as possible: functional representation of the spectra, spectral smoothing and penalised regression ${ }^{16}$. The utility of each technique was considered independently as well as in conjunction with one another. Representing the spectra with a set of basis functions of size $k$, written as a proportion of the total number of spectral variables, both removes excess noise from the data and increases computational efficiency by reducing the dimensionality of the data. Spectral smoothing achieves a similar effect but through the use of B-spline functions and with no reduction in dimensionality. Finally, ridge regression, a form of penalisation with a squared penalty term, shrinks the values of the coefficient function and favours models with lower numbers of explanatory variables. 
The number of observations belonging to each class was often imbalanced so the training and independent testing data were sampled from to enforce the same number of observations from each class and optimise the model's performance both within- and out-of-sample. The balanced training data was further split into training, cross-validation and testing subsets of sizes 50\%, $25 \%$ and $25 \%$ respectively. This process was repeated 50 times to minimise the possibility of sampling error from both the balancing and data splitting. For each of the 50 iterations multiple sub-models were fit using the training subset, (with 2-20 components), and for those models deploying penalised regression, with 10 exponentially increasing values of the penalty parameter from 0.01 to 20 . The accuracy of each was tested using the cross-validation subset to determine which option maximised the area under the receiver operating characteristic curve (AUC, value closer to 1 indicating better performance). Once the maximal sub-model were identified, the submodel with a lesser number of components with AUC value within $\tau$ of the maximised AUC value was selected as the overall optimal model that is presented in the results. By applying this finalised model to the testing subset, the critical threshold minimising the error arising from classifying these mosquitoes as infectious or uninfectious was estimated. The error structure was calculated as the number of false negative and false positive predictions divided by the total number of observations in this subset. The overall model error is taken as the average of the 50 models to the 50 testing subsets (within-sample-error) or to the independent test set using mosquitoes infected in a different location and not used in model training or validation (out-ofsample error). Note that this within-sampling error is more rigorous than other reported out-ofsampling methods which jack-knife data (exclude one sample from the training set each time, and test accuracy on that sample). Sixty-four different parameter combinations were explored for each experiment using a grid search approach in which each of the three smoothing techniques above were considered as binary variables (with 0 implying exclusion and 1 inclusion) with four tuning parameter values tau $=0.05,0.1,0.15,0.2$ and three basis sizes $\mathrm{k}=25 \%, 50 \%, 75 \%$ for those models deploying functional representation. The optimal model was then selected by considering the parameter combination producing the minimal overall error in conjunction with those with minimal bias, measured by the absolute value between the false positive and false negative rates. All analyses were carried out in $\mathrm{R}$ using the package mlevcm ${ }^{16}$ and assume that diagnostics (microscopy and PCR) are $100 \%$ accurate. 


\section{References}

1. WORLD HEALTH ORGANIZATION. WORLD MALARIA REPORT 2019. (WORLD HEALTH ORGANIZATION, 2019).

2. Paixão, E. S., Teixeira, M. G. \& Rodrigues, L. C. Zika, chikungunya and dengue: the causes and threats of new and re-emerging arboviral diseases. BMJ Glob Health 3, e000530 (2018).

3. Bhatt, S. et al. The effect of malaria control on Plasmodium falciparum in Africa between 2000 and 2015. Nature 526, 207-211 (2015).

4. WHO. Training module on malaria control: Malaria entomology and vector control. Guide for participants. 190 (2013).

5. Burkot, T. R., Williams, J. L. \& Schneider, I. Identification of Plasmodium FalciparumInfected Mosquitoes by a Double Antibody Enzyme-Linked Immunosorbent Assay *. The American Journal of Tropical Medicine and Hygiene 33, 783-788 (1984).

6. Stone, W. et al. A comparison of Plasmodium falciparum circumsporozoite protein-based slot blot and ELISA immuno-assays for oocyst detection in mosquito homogenates. Malar J 14, 451 (2015).

7. Tassanakajon, A., Boonsaeng, V., Wilairat, P. \& Panyim, S. Polymerase chain reaction detection of Plasmodium falciparum in mosquitoes. Transactions of the Royal Society of Tropical Medicine and Hygiene 87, 273-275 (1993).

8. Esperança, P. M., Blagborough, A. M., Da, D. F., Dowell, F. E. \& Churcher, T. S. Detection of Plasmodium berghei infected Anopheles stephensi using near-infrared spectroscopy. Parasites Vectors 11, 377 (2018).

9. Maia, M. F. et al. Detection of malaria in insectary-reared Anopheles gambiae using nearinfrared spectroscopy. http://biorxiv.org/lookup/doi/10.1101/533802 (2019) doi:10.1101/533802. 
10. Fernandes, J. N. et al. Rapid, noninvasive detection of Zika virus in Aedes aegypti mosquitoes by near-infrared spectroscopy. Sci. Adv. 4, eaat0496 (2018).

11. Sikulu-Lord, M. T. et al. Rapid and Non-destructive Detection and Identification of Two Strains of Wolbachia in Aedes aegypti by Near-Infrared Spectroscopy. PLoS Negl Trop Dis 10, e0004759 (2016).

12. Ong, O. T. W. et al. Ability of near-infrared spectroscopy and chemometrics to predict the age of mosquitoes reared under different conditions. Parasites Vectors 13, 160 (2020).

13. Bompard, A. et al. High Plasmodium infection intensity in naturally infected malaria vectors in Africa. International Journal for Parasitology S002075192030182X (2020) doi:10.1016/j.ijpara.2020.05.012.

14. Mayagaya, V. S. et al. Non-destructive Determination of Age and Species of Anopheles gambiae s.1. Using Near-infrared Spectroscopy. The American Journal of Tropical Medicine and Hygiene 81, 622-630 (2009).

15. Sikulu, M. et al. SNhoertarerp-oirnt frared spectroscopy as a complementary age grading and species identification tool for African malaria vectors. 7 (2010).

16. Esperança, P. M., Da, D. F., Lambert, B., Dabiré, R. K. \& Churcher, T. S. Functional data analysis techniques to improve the generalizability of near-infrared spectral data for monitoring mosquito populations. http://biorxiv.org/lookup/doi/10.1101/2020.04.28.058495 (2020) doi:10.1101/2020.04.28.058495.

17. Alex K. Musiime. Is that a real oocyst? Insectary establishment and identification of Plasmodium falciparum oocysts in midguts of Anopheles mosquitoes fed on infected human blood in Tororo, Uganda. (2019). 
18. Milali, M. P. et al. Age grading An. gambiae and An. arabiensis using near infrared spectra and artificial neural networks. PLoS ONE 14, e0209451 (2019).

19. Milali, M. P. et al. An Autoencoder and Artificial Neural Network-based Method to Estimate Parity Status of Wild Mosquitoes from Near-infrared Spectra. http://biorxiv.org/lookup/doi/10.1101/2020.01.25.919878 (2020) doi:10.1101/2020.01.25.919878.

20. Mwanga, E. P. et al. Using mid-infrared spectroscopy and supervised machine-learning to identify vertebrate blood meals in the malaria vector, Anopheles arabiensis. Malar J 18, 187 (2019).

21. González-Jiménez, M. et al. Prediction of malaria mosquito species and population age structure using mid-infrared spectroscopy and supervised machine learning. http://biorxiv.org/lookup/doi/10.1101/414342 (2018) doi:10.1101/414342.

22. Boissière, A. et al. Application of a qPCR Assay in the Investigation of Susceptibility to Malaria Infection of the M and S Molecular Forms of An. gambiae s.s. in Cameroon. PLoS ONE 8, e54820 (2013).

23. Sangare, I. et al. Studying fitness cost of Plasmodium falciparum infection in malaria vectors: validation of an appropriate negative control. Malar J 12, 2 (2013).

24. Anthony, T. G., Trueman, H. E., Harbach, R. E. \& Vogler, A. P. Polymorphic microsatellite markers identified in individual Plasmodium falciparum oocysts from wild-caught Anopheles mosquitoes. Parasitology 121, 121-126 (2000).

25. Santolamazza, F. et al. Insertion polymorphisms of SINE200 retrotransposons within speciation islands of Anopheles gambiae molecular forms. Malar J 7, 163 (2008). 
26. Sikulu, M. et al. Evaluating RNAlater ${ }^{\circledR}$ as a preservative for using near-infrared spectroscopy to predict Anopheles gambiae age and species. Malar J 10, 186 (2011).

\section{Acknowledgments:}

Funding: The work was supported by UK Medical Research Council (MRC) Project Grant (MR/P01111X/1) and the MRC / UK Department for International Development (DFID) under the MRC/DFID Concordat agreement.

AMB thanks the MRC (MR/N00227X/1)) Isaac Newton Trust, Alborada Fund, Wellcome Trust ISSF and University of Cambridge JRG Scheme, GHIT and the Royal Society for funding.

Author contributions: DFD, RKD, TL, KM and TSC conceived the study, DFD and MBS conducted the spectroscopy, laboratory and field work, DFD, MBS, SRY, KS, JB and AMB conducted the molecular analysis, RM, FD, PME and TSC were responsible for the machine learning and all authors approved the final manuscript.

Competing interests: None

Data and materials availability: All data will be placed on an online repository once manuscript is accepted. 


\section{Figures}
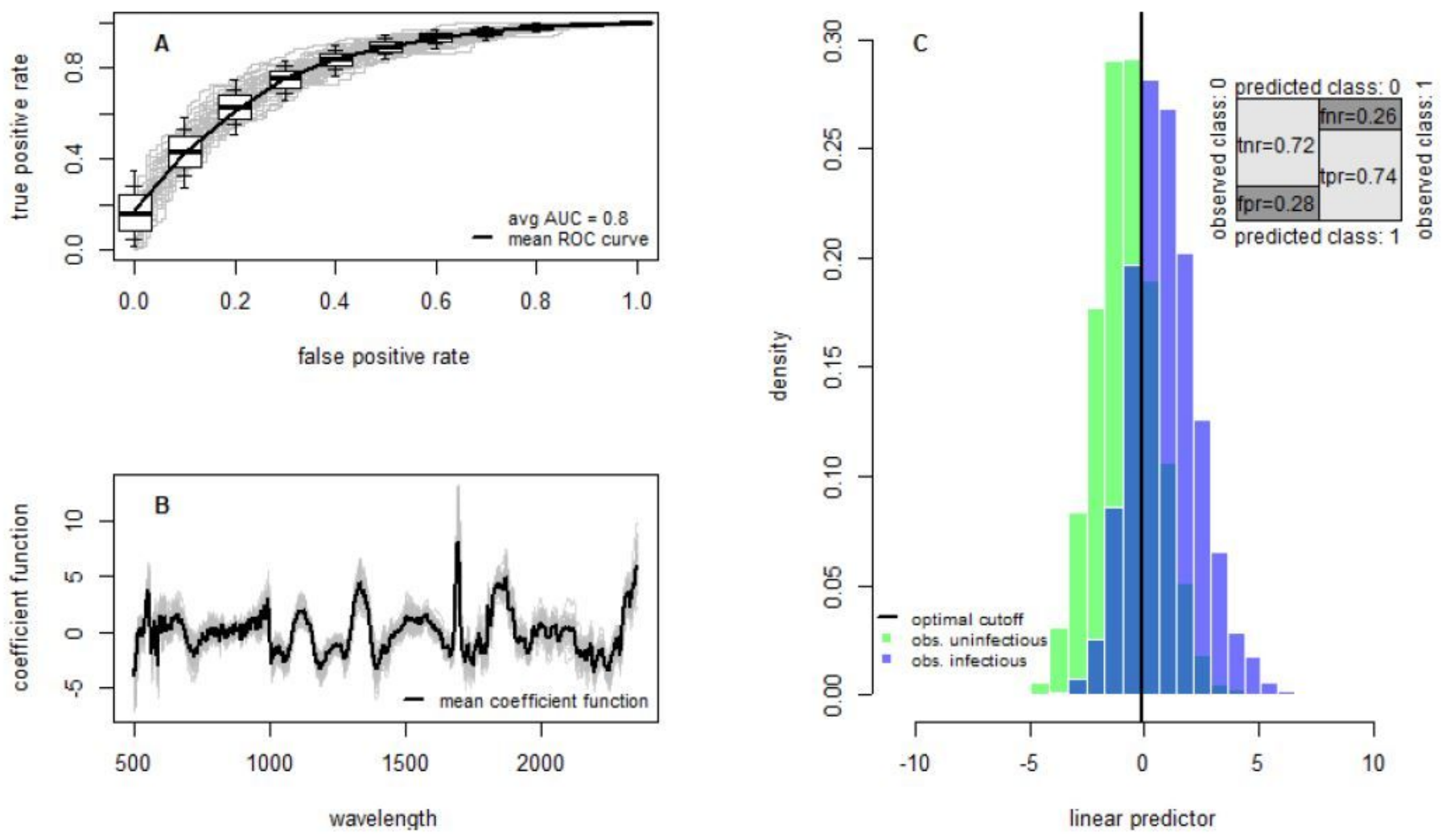

\section{Figure 1}

The ability of NIRS to predict laboratory-reared mosquitoes infectious with wild parasites. All models were trained on sporozoite positive and sporozoite negative laboratory reared mosquitoes using all the data presented in Table 1. (A) The receiver operating characteristic (ROC) curve for the best-fit model showing the false positive and true positive rates achievable for different classification probability thresholds whilst the overall performance is given by the average area under the ROC curve (AUC). A perfect model with $100 \%$ sensitivity and specificity would be in the top left corner (coordinates 0,1 ). Solid line shows the average ROC curve with boxplots showing the variability for 50 randomizations of the training, validation and testing datasets (with box edges, inner and outer whiskers showing 25th/75th, 15th/85th and 5th/95th percentiles, respectively; and the black line inside the box showing the median/50thpercentile). (B) Coefficient functions for the best fit model for each of the 50 dataset randomizations (grey lines) and the corresponding average (black line). (C) The histogram of the estimated linear predictor for the test mosquitoes, color of the bars indicates the true class, shows the model's ability to separate the two groups of mosquitoes. The vertical black line indicates the optimum threshold for classifying mosquitoes as infectious or not. The shaded area where the two distributions overlap corresponds to misclassified test observations - false negatives to the left and false positives to the right of the optimal classification threshold. The confusion matrix (inset) shows the different error rates: tnr, 
true negative rate (specificity); fnr, false negative rate; $\mathrm{fpr}$, false positive rate; and tpr, true positive rate (sensitivity).
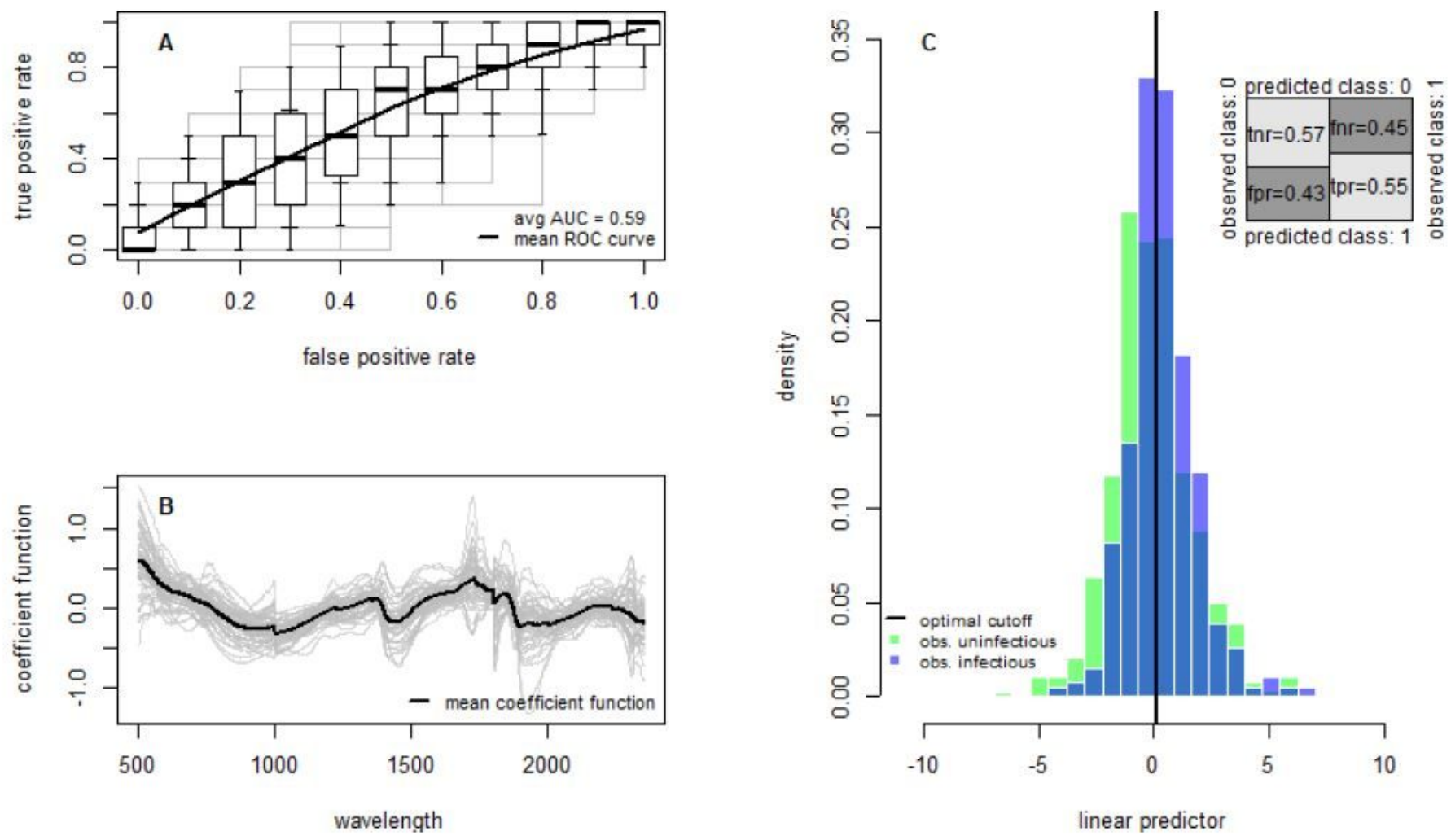

Figure 2

The ability of NIRS to predict field caught mosquitoes with high number of sporozoites. All models were trained using mosquitoes infected in the wild and were either sporozoite positive mosquitoes with $>20$ sporozoite per Anopheles (20 gene copy number as defined by qPCR) or sporozoite negative mosquitoes (Table 1) (A) The ROC curve for the best-fit model showing the false positive and true positive rates achievable for different classification probability thresholds whilst the overall performance is given by the AUC. Solid line shows the average ROC curve with boxplots showing the variability for 50 randomizations of the training, validation and testing datasets (with box edges, inner and outer whiskers showing 25th/75th, 15th/85th and 5th/95th percentiles, respectively; and the black line inside the box showing the median/50th-percentile). (B) Coefficient functions for the best fit model for each of the 50 dataset randomizations (grey lines) and the corresponding average (black line). (C) The histogram of the estimated linear predictor for the test mosquitoes, color of the bars indicates the true class, shows the model's ability to separate the two groups of mosquitoes. The vertical black line indicates the optimum threshold for classifying mosquitoes as infectious or not. The shaded area where the two distributions overlap corresponds to misclassified test observations - false negatives to the left and false positives to the right of the optimal classification threshold. The confusion matrix (inset) shows the different error 
rates: $t n r$, true negative rate (specificity); fnr, false negative rate; $f p r$, false positive rate; and tpr, true positive rate (sensitivity).

\section{Supplementary Files}

This is a list of supplementary files associated with this preprint. Click to download.

- NIRSPfSupplementaryfileSR.pdf 